\title{
Intramolecular dynamics of linear macromolecules by fluorescence correlation spectroscopy
}

\author{
R. G. Winkler* \\ Institut für Festkörperforschung, Forschungszentrum Jülich, D-52425 Jülich, Germany
}

S. Keller and J. O. Rädler

Ludwig-Maximilians-Universität, Geschwister-Scholl-Platz, 1, D-80539 München, Germany

(Received 2 November 2004; revised manuscript received 22 December 2005; published 17 April 2006)

\begin{abstract}
A theoretical description of the dynamics of DNA molecules and actin filaments in solution as measured experimentally by fluorescence correlation spectroscopy is provided and compared to recent experimental results. Particular attention is paid to the contribution of the intramolecular dynamics to the fluorescence correlation function. Using a semiflexible chain model, a theoretical expression is presented for the fluorescence correlation spectroscopy correlation function. The dependence of this function on various model parameters, such as chain length, persistence length, and fluorescence label density, is discussed. Our investigations show that the intramolecular dynamics provides a significant contribution or even dominates the correlation function as soon as the longest intramolecular relaxation time significantly exceeds the shortest experimentally accessible time. Correspondingly, the shape of the correlation function changes considerably. Approximate analytical expressions are provided, which are in qualitative agreement with the exact theoretical solutions as well as experimental results, for both DNA and actin filaments. Our approach is in agreement with the predictions of the Zimm model, in the limit of very flexible polymers, as well as the predictions of semiflexible polymer models with respect to the intramolecular dynamics in solution.
\end{abstract}

DOI: 10.1103/PhysRevE.73.041919

PACS number(s): 87.15.He, 87.15.Ya, 83.80.Rs

\section{INTRODUCTION}

Fluorescence correlation spectroscopy (FCS) is today one of the most powerful and sensitive techniques to study the dynamics of labeled monomolecules in solution [1-8]. In FCS, one detects the fluctuations of the fluorescent light intensity in a small and fixed volume element, usually formed by a laser focus of submicron size. The temporal autocorrelation of these fluctuations can be related to the dynamical processes involved, such as diffusion behavior, reaction kinetics, or photophysical processes. Thus, with the appropriate model of the particular system dynamics, different characteristic rates can be measured.

FCS has been applied in studies of the diffusive dynamics of simple colloids [9], polymers [10-12], and biomolecules [13-16]. For the first time, molecular mobility can be measured at a molecular level in various cellular compartments. In particular, translational diffusion and active transport can be distinguished [17], and differences in the local environment can be studied by alterations in the molecular mobility of known fluorophores [18]. Simultaneous measurements of mobility parameters and association or dissociation kinetics suggests that FCS might be a useful technique to gain insight into mechanisms and pathways of signal transduction [19-21]. In most of the studies, the size of the diffusive object is smaller than the focal volume, even when polymers are studied [10-12]. With increasing interest in colloidal systems, extensions have been proposed to diffusion of particles with sizes larger than the focal volume [22]. A more complex dynamic arises when biological macromolecules are investi-

*Electronic address: r.winkler@fz-juelich.de gated that are both significantly larger than the laser focus and labeled with more than one chromophore [16]. For such objects, the FCS correlation function is determined by the diffusion of the whole molecule as well as the intramolecular dynamics. To separate the corresponding contributions requires an adequate theoretical description of both the molecular dynamics as well as the FCS correlation function.

Biological macromolecules exhibit a broad spectrum of conformational degrees of freedom ranging from very flexible molecules (e.g., ss-DNA), semiflexible molecules (e.g., ds-DNA, actin filament), to rodlike objects (e.g., microtubule). The stiffness and relaxation behavior are essential for their biological functions. The equilibrium properties of semiflexible polymers are mostly elucidated either in terms of the Kratky-Porod model [23,24] or a semiflexible Gaussian chain model $[25,26]$ (and references therein). Most of the theoretical investigations of the dynamics of flexible macromolecules are based on the Rouse-Zimm model [27-31]. However, the persistence length of biological molecules is often on the order of or even exceeds the size of the laser focus in an FCS experiment. The real molecule is very different from a Rouse chain on this length scale due to chain stiffness. As long as the biological molecule is rather rodlike, its dynamics can be described by the Kratky-Porod wormlike chain model [32-34]. A model that accounts for the dynamics of polymers of any stiffness has been presented in Refs. [35-37]. The major advantage of this approach is that it allows us to analytically investigate the dynamics of semiflexible macromolecules, both in solution as well as in melt. The full dynamic structure factor can be obtained. In contrast, many other approaches rely on the first cumulant of the dynamic structure factor extracted from equilibrium properties. In particular, a detailed comparison of theoretical results to quasielastic neutron and dynamic light-scattering experi- 
ments on various natural and synthetic macromolecules in dilute solution exhibits good agreement [36]. Similar agreement has been obtained for the coherent and incoherent dynamic structure factors of various macromolecules in melt $[38,39]$. Moreover, the intramolecular dynamics of partially stretched DNA molecules can be very well described in agreement with experiments [40].

In this paper, we theoretically determine the FCS correlation function of semiflexible macromolecules and compare the results to experimental measurements on DNA molecules and actin filaments. The dependence of the correlation function on polymer length for DNA and actin filaments is discussed, as well as its theoretical dependence on the label density. Particular attention is paid to the contribution of the intramolecular dynamics to the FCS correlation function.

The paper is organized as follows. In Sec. II, the theoretical background of the fluorescence correlation technique is described. The theoretical model is introduced, its equilibrium properties, and the solutions of the equations of motion are discussed briefly. The FCS correlation function is calculated using the dynamic structure factor. In Sec. III, the experimental equipment is described. Results are presented in Sec. IV. Section V summarizes our findings.

\section{THEORETICAL BACKGROUND}

\section{A. Fluorescence correlation spectroscopy}

The principle of FCS was invented and realized in the early 1970s by Magde et al. [41], Elson and Magde [42], and Magde et al. [43]. By introducing a confocal setup to detect the emitted light, the sensitivity of the method was enormously enhanced [44]. Under carefully chosen conditions [45], the observation volume can be approximated by a three-dimensional Gaussian ellipsoid

$$
W(\boldsymbol{r})=W_{0} \exp \left(-2 \frac{x^{2}+y^{2}}{r_{0}^{2}}\right) \exp \left(-2 \frac{z^{2}}{z_{0}^{2}}\right)
$$

This function represents the probability of exciting and detecting a fluorescent molecule at position $\boldsymbol{r}$ in sample space. The $1 / e^{2}$-radius $r_{0}$ is the waist size of the focused excitation laser; the value $z_{0}$ is mainly determined by the confocal detection optics.

The time-dependent intensity

$$
I(t)=\int W(\boldsymbol{r}) C(\boldsymbol{r}, t) d^{3} x
$$

in a FCS experiment (often measured with an APD) therefore depends both on the observation volume given by the setup and the dynamic properties of the system under study. $C(\boldsymbol{r}, t)$ is the time- and space-dependent density of fluorescent markers. Rička and Binkert have shown that a transformation to Fourier space simplifies the theoretical calculation of the autocorrelation function [46]. It can be written as

$$
g_{\mathrm{D}}(t)=\frac{\left\langle\delta I\left(t^{\prime}\right) \delta I\left(t^{\prime}+t\right)\right\rangle_{t^{\prime}}}{\left\langle I\left(t^{\prime}\right)\right\rangle_{t^{\prime}}^{2}}=g_{\mathrm{D}}(0) \frac{\int \Omega(\boldsymbol{q}) \tilde{C}(\boldsymbol{q}, t) d^{3} q}{\int \Omega(\boldsymbol{q}) \tilde{C}(\boldsymbol{q}, 0) d^{3} q},
$$

where $\Omega(\boldsymbol{q})=\mathrm{FT}\{W(r)\}_{\boldsymbol{q}} \mathrm{FT}\{W(\boldsymbol{r})\}_{-\boldsymbol{q}}$ is a Gaussian filter function in Fourier space representing the observation volume. Using Eq. (1), $\Omega$ explicitly reads

$$
\Omega(\boldsymbol{q})=\Omega_{0} \exp \left(-\frac{r_{0}^{2}}{4}\left(q_{x}^{2}+q_{y}^{2}\right)-\frac{z_{0}^{2}}{4} q_{z}^{2}\right) .
$$

(Note, the constant $\Omega_{0}$ drops in the calculation of $g_{\mathrm{D}}$.) $\widetilde{C}(\boldsymbol{q}, t)$ is the fluorescence density-density autocorrelation function in Fourier space. $g_{\mathrm{D}}(0)=1 /\left(\bar{C} V_{\text {eff }}\right)$ with $\bar{C}$ the mean particle concentration and $V_{\text {eff }}=\pi^{3 / 2} r_{0}^{2} z_{0}$ the so-called effective volume. The FCS correlation function (3) contains information about diffusion, possible chemical reactions, and the photophysics of the molecules.

Assuming pointlike scattering centers, the correlation function can be written as $C(\boldsymbol{r}, t)=\sum_{i=1}^{N} b_{i} \delta\left[\boldsymbol{r}-\boldsymbol{r}_{i}(t)\right]$, where $b_{i}$ is the scattering amplitude of particle $i$. The autocorrelation function $\widetilde{C}(\boldsymbol{q}, t)$ is then equal to the dynamic structure factor $S(\boldsymbol{q}, t)$.

For the simplest case of monodisperse Brownian diffusing objects with attached pointlike dyes, the solution of the diffusion equation leads directly to $S(\boldsymbol{q}, t)=\exp \left(-q^{2} D t\right)$, where $D$ is the diffusion coefficient. Integration of Eq. (3) with the filter function (4) yields

$$
G_{\mathrm{D}}(t)=\frac{g_{\mathrm{D}}(t)}{g_{\mathrm{D}}(0)}=\left(1+\frac{t}{\tau}\right)^{-1}\left(1+\frac{r_{0}^{2}}{z_{0}^{2}} \frac{t}{\tau}\right)^{-1 / 2},
$$

which describes the diffusional decay of the correlation function, where $\tau=r_{0}^{2} / 4 D$ is the diffusion time. By fitting this function to experimental correlation data, the diffusion coefficient and the concentration can be determined if the observation volume is well characterized.

In the case of a fractional time dependence, i.e., $S(\boldsymbol{q}, t)$ $=\exp \left[-q^{2}(\Gamma t)^{\beta}\right]$, as observed in anomalous diffusion or polymer dynamics [30], Eq. (5) changes to

$$
G_{\mathrm{D}}=\left[1+\left(\frac{t}{\tau^{*}}\right)^{\beta}\right]^{-1}\left[1+\frac{r_{0}^{2}}{z_{0}^{2}}\left(\frac{t}{\tau^{*}}\right)^{\beta}\right]^{-1 / 2},
$$

with $\tau^{*}=\left(r_{0}^{2} / 4 \Gamma\right)^{1 / \beta}$ [16]. Equations (5) and (6) demonstrate that the time dependence of the correlation function is determined by the dynamic structure factor. Therefore, an adequate dynamic structure factor is needed to extract molecular parameters from the correlation function. One aspect of the present paper is to determine the correlation function for a semiflexible polymer and hence advance the analysis presented in Ref. [16].

FCS is not only sensitive to intensity fluctuations due to diffusion but also due to photokinetic processes of the fluorescent dyes. Changes in the brightness of the diffusing object can be detected with FCS as long as they are signifi- 
cantly faster than the diffusion time. The most prominent and well-known process is the triplet decay. The excited molecules can occupy a metastable dark triplet state. This leads to intensity fluctuations in the time range of this lifetime. The correlation function of the triplet state kinetics can be approximated by an exponential decay [47]

$$
G_{\text {Triplet }}=1+\frac{T}{1-T} \exp \left(-\frac{t}{\tau_{\mathrm{T}}}\right) .
$$

$T$ denotes the fraction of dyes in the triplet state and $\tau_{\mathrm{T}}$ is the triplet time.

For objects carrying several chromophores, the situation is more complex. A chain with $M$ dyes along its contour can have $M+1$ different states of brightness, depending on the number of dyes in a dark state. The kinetics of the fluctuations can be modeled with an $M+1$ isomerization, that means by switching on or off one single dye the state of the chain changes to the neighboring state. Assuming constant on and off rates $k_{\text {on }}$ and $k_{\text {off }}$ between the different states, the problem reduces to a finite death and birth process. The solution of this problem has been presented before [16]. We take into account these processes by introducing the function $G_{\text {Iso }}(t)$, which depends only on the number of dyes along the chain and the two rates $k_{\text {on }}$ and $k_{\text {off }}$. The function is a superposition of $M+1$ exponentially decaying functions with decay rates and amplitudes given by the on and off rates and the starting conditions. The characteristic decay time of $G_{\text {Iso }}(t)$ turned out to be $10-100 \mu$ s. As the triplet state kinetics is faster and the diffusion process is slower, this function describes an intermediate decay.

The time scales of the kinetic processes are well separated. Thus, the correlation function can be written as the product of the respective functions [48]. In our case, this results in

$$
g(t)=g_{\mathrm{D}}(0) G_{\text {Triplet }}(t) G_{\text {Iso }}(t) G_{\mathrm{D}}(t) .
$$

The functions $G_{\text {Triplet }}(t)$ and $G_{\text {Iso }}(t)$ are both normalized to decay to the value 1 for large times. $G_{\mathrm{D}}(t)$ decays from 1 to 0 . To investigate the diffusional decay, it is important to get the amplitude of the correlation function. The experimental correlation functions were fitted with Eqs. (6) and (8). Then the experimental data were normalized by dividing them by the amplitude, yielding a new function

$$
G(t)=g(t) / g_{\mathrm{D}}(0)=G_{\text {Triplet }}(t) G_{\text {Iso }}(t) G_{\mathrm{D}}(t) .
$$

The amplitude of this function is normalized in such a way that the diffusion part decays from 1 to 0 (see also Ref. [16]). To gain insight into the FCS correlation function of a polymer, we briefly discuss the dynamical properties of semiflexible chain molecules in dilute solution.

\section{B. Equilibrium properties of semiflexible polymers}

The polymer chain is modeled as a continuous, differential space curve $\boldsymbol{r}(s)$, where $s(-L / 2 \leqslant s \leqslant L / 2)$ is the contour coordinate along the chain. A minimal model for a semiflexible polymer is a Gaussian semiflexible chain [25]. Its basic features are as follows: (i) The chain is composed of Gauss- ian segments, i.e., the distribution function of the distance between two points along the chain contour is Gaussian; and (ii) bending restrictions are taken into account. The distribution and partition function of such a chain can be systematically derived by the maximum entropy principle $[25,49]$. For a continuous chain of length $L$, the partition function reads

$$
\begin{aligned}
Z= & \int \exp \left\{-\nu \int_{-L / 2}^{L / 2}\left(\frac{\partial \boldsymbol{r}}{\partial s}\right)^{2} d s-\frac{\epsilon}{2} \int_{-L / 2}^{L / 2}\left(\frac{\partial^{2} \boldsymbol{r}}{\partial s^{2}}\right)^{2} d s\right. \\
& \left.-\nu_{0}\left[\left(\frac{\partial \boldsymbol{r}(-L / 2)}{\partial s}\right)^{2}+\left(\frac{\partial \boldsymbol{r}(L / 2)}{\partial s}\right)^{2}\right]\right\} \mathcal{D}^{3} x
\end{aligned}
$$

The Lagrangian multipliers $\nu, \nu_{0}$, and $\epsilon$ are given by

$$
\nu=\frac{3 p}{2}, \quad \nu_{0}=\frac{3}{4}, \quad \epsilon=\frac{3}{4 p}, \quad p=\frac{1}{2 l_{p}} .
$$

The terms with the first derivative in $r$ capture the chain flexibility, i.e., they account for the chain entropy. The term with the second derivative is the bending energy. The chain ends behave differently from the rest of the chain, which is captured in the terms with $\nu_{0}$. The major difference to the Kratky-Porod wormlike chain model [23] is the constraint for the tangent vector $\boldsymbol{u}=\partial \boldsymbol{r}(s) / \partial s$. In the Gaussian model, the constraint $\boldsymbol{u}^{2}(s)=1$ is relaxed to $\left\langle\boldsymbol{u}^{2}(s)\right\rangle=1$.

An oft-required quantity is the joint probability distribution $\Psi\left[\boldsymbol{r}(s), \boldsymbol{r}\left(s^{\prime}\right)\right]$ of two points at $\boldsymbol{r}(s)$ and $\boldsymbol{r}\left(s^{\prime}\right)$. A straightforward calculation yields

$$
\begin{aligned}
\Psi\left[\boldsymbol{r}(s)-\boldsymbol{r}\left(s^{\prime}\right)\right]= & \left(\frac{3}{2 \pi \sigma\left(s-s^{\prime}\right)}\right)^{3 / 2} \\
& \times \exp \left(-\frac{3\left[\boldsymbol{r}(s)-\boldsymbol{r}\left(s^{\prime}\right)\right]^{2}}{2 \sigma\left(s-s^{\prime}\right)}\right),
\end{aligned}
$$

with

$$
\sigma\left(s-s^{\prime}\right)=\left\langle\left[\boldsymbol{r}(s)-\boldsymbol{r}\left(s^{\prime}\right)\right]^{2}\right\rangle=\frac{\left|s-s^{\prime}\right|}{p}-\frac{1}{2 p^{2}}\left(1-e^{-2 p\left|s-s^{\prime}\right|}\right) .
$$

As a particular result, the mean square end-to-end distance is obtained from Eq. (13) by setting $s=L / 2$ and $s^{\prime}=-L / 2$. Moreover, the radius of gyration is given by [25]

$$
\left\langle r_{g}^{2}\right\rangle=\frac{L}{6 p}-\frac{1}{4 p^{2}}+\frac{1}{4 p^{3} L}-\frac{1}{8 p^{4} L^{2}}\left(1-e^{-2 p L}\right) .
$$

\section{Dynamics of semiflexible polymers}

To describe the dynamics of macromolecules in dilute solution, hydrodynamic interactions $[30,36]$ mediated by the solvent particles have to be taken into account. This is achieved by the Rotne-Prager tensor $\mathbf{Q}\left(\boldsymbol{r}(s), \boldsymbol{r}\left(s^{\prime}\right)\right)[36,50]$, which has been shown to be a first-order correction to the Oseen tensor [51].

In order to arrive at an analytically tractable equation of motion, we apply the preaveraging approximation by Zimm [29]. This approximation replaces the hydrodynamic tensor 
by its average calculated with the equilibrium distribution function. With the joint probability distribution of a Gaussian semiflexible chain (12), the averaged hydrodynamic tensor is given by $\mathbf{H}\left(s, s^{\prime}\right)=\mathbf{I}\left[\delta\left(s-s^{\prime}\right) / 3 \pi \eta+Q\left(s-s^{\prime}\right)\right]$, where the $\delta$ function accounts for the local friction, $\eta$ is the solvent viscosity, and

$$
\begin{aligned}
Q\left(s-s^{\prime}\right)= & \frac{\Theta\left(\left|s-s^{\prime}\right|-d\right)}{3 \pi \eta} \sqrt{\frac{3}{2 \pi \sigma\left(s-s^{\prime}\right)}} \\
& \times \exp \left(-\frac{3 d^{2}}{2 \sigma\left(s-s^{\prime}\right)}\right) .
\end{aligned}
$$

Here, the Heaviside step function $\Theta$ is introduced to exclude self-interactions and $d$ is the thickness of the molecule.

The equation of motion of the polymer chain including hydrodynamic interactions is then given by the Langevin equation [36]

$$
\begin{aligned}
\frac{\partial}{\partial t} \boldsymbol{r}(s, t)= & \int_{-L / 2}^{L / 2} \mathbf{H}\left(s, s^{\prime}\right)\left[2 \nu k_{B} T \frac{\partial^{2}}{\partial s^{\prime 2}} \boldsymbol{r}\left(s^{\prime}, t\right)\right. \\
& \left.-\epsilon k_{B} T \frac{\partial^{4}}{\partial s^{\prime 4}} \boldsymbol{r}\left(s^{\prime}, t\right)+\boldsymbol{\Gamma}\left(s^{\prime}, t\right)\right] d s^{\prime},
\end{aligned}
$$

with the boundary conditions

$$
\begin{gathered}
{\left[2 \nu \frac{\partial}{\partial s} \boldsymbol{r}(s, t)-\epsilon \frac{\partial^{3}}{\partial s^{3}} \boldsymbol{r}(s, t)\right]_{ \pm L / 2}=0,} \\
{\left[2 \nu_{0} \frac{\partial}{\partial s} \boldsymbol{r}(s, t) \pm \epsilon \frac{\partial^{2}}{\partial s^{2}} \boldsymbol{r}(s, t)\right]_{ \pm L / 2}=0 .}
\end{gathered}
$$

The stochastic force $\boldsymbol{\Gamma}(s, t)$ is assumed to be stationary, Markovian, and Gaussian with zero mean [30].

To solve the equation of motion, we use an eigenfunction expansion in terms of the eigenfunctions of the eigenvalue equation

$$
\epsilon k_{B} T \frac{d^{4}}{d s^{4}} \psi_{n}(s)-2 \nu k_{B} T \frac{d^{2}}{d s^{2}} \psi_{n}(s)=\xi_{n} \psi_{n}(s) .
$$

The eigenfunctions are given by

$$
\begin{gathered}
\psi_{0}=\sqrt{\frac{1}{L}}, \\
\psi_{n}(s)=\sqrt{\frac{c_{n}}{L}}\left(\zeta_{n}^{\prime} \frac{\sinh \zeta_{n}^{\prime} s}{\cosh \zeta_{n}^{\prime} L / 2}+\zeta_{n} \frac{\sin \zeta_{n} s}{\cos \zeta_{n} L / 2}\right), \quad \forall n \text { odd }, \\
\psi_{n}(s)=\sqrt{\frac{c_{n}}{L}}\left(\zeta_{n}^{\prime} \frac{\cosh \zeta_{n}^{\prime} s}{\sinh \zeta_{n}^{\prime} L / 2}-\zeta_{n} \frac{\cos \zeta_{n} s}{\sin \zeta_{n} L / 2}\right), \quad \forall n \text { even. }
\end{gathered}
$$

The $c_{n}$ s follow from the normalization condition. The wave numbers $\zeta_{n}$ and $\zeta_{n}^{\prime}$, where $\zeta_{n}^{\prime 2}-\zeta_{n}^{2}=4 p^{2}$, are determined by the boundary conditions (17) and (18). $\psi_{0}$ describes the translational motion of the whole molecule.

To solve Eq. (16), the eigenfunction expansions

$$
\boldsymbol{r}(s, t)=\sum_{n=0}^{\infty} \boldsymbol{\chi}_{n}(t) \psi_{n}(s), \quad \boldsymbol{\Gamma}(s, t)=\sum_{n=0}^{\infty} \boldsymbol{\Gamma}_{n}(t) \psi_{n}(s)
$$

are inserted, which yields

$$
\frac{\partial}{\partial t} \boldsymbol{\chi}_{n}(t)=\sum_{m=0}^{\infty}\left(Q_{n m}+\frac{\delta_{n m}}{3 \pi \eta}\right)\left[-\frac{3 \pi \eta}{\tau_{m}} \boldsymbol{\chi}_{m}(t)+\boldsymbol{\Gamma}_{m}(t)\right] .
$$

The $\tau_{n} \mathrm{~s}$ are the relaxation times in the free draining limit and are related to the eigenvalues $\xi_{n}$ via $\tau_{n}=\gamma / \xi_{n}$, where $\gamma$ $=3 \pi \eta$ is the friction constant per length. The $Q_{n m} \mathrm{~s}$ are the matrix elements of $Q\left(s-s^{\prime}\right)$ in terms of the eigenfunctions $\psi_{n}(s)$.

A numerical calculation shows that the interaction matrix $Q_{n m}$ is almost diagonal over the whole range of the flexibility parameter $p L$. Hence, except for the first mode $(n=1)$ in the limit of large stiffness, the interaction matrix is well approximated by

$$
Q_{n m}=\sqrt{\frac{2}{3 \pi^{3}}} \frac{\delta_{n m}}{\eta L} \int_{d}^{L} \frac{(L-s)}{\sqrt{\sigma(s)}} \exp \left(-\frac{3 d^{2}}{2 \sigma(s)}\right) \cos \zeta_{n} s d s .
$$

For $n=1$ and $p L \rightarrow 0$, the full expression for $Q_{11}$ has to be used [35]. The amplitudes $\boldsymbol{\chi}_{n}(t)$ are then given by

$$
\boldsymbol{\chi}_{n}(t)=\frac{\tau_{n}}{3 \pi \eta \tilde{\tau}_{n}} \int_{-\infty}^{t} e^{-\left(t-t^{\prime}\right) / \tilde{\tau}_{n}} \boldsymbol{\Gamma}_{n}\left(t^{\prime}\right) d t^{\prime},
$$

with the relaxation times

$$
\widetilde{\tau}_{n}=\frac{\tau_{n}}{1+3 \pi \eta Q_{n n}} .
$$

The time correlation functions of appropriate polymer properties are often required when the above results are applied to experimental measurements. Many of these correlation functions can simply be obtained from the correlation functions of the amplitudes $\boldsymbol{\chi}_{n}$. A straightforward calculation yields

$$
\left\langle\boldsymbol{\chi}_{n}(t) \boldsymbol{\chi}_{m}(0)\right\rangle=\frac{k_{B} T}{\pi \eta} \tau_{n} \delta_{n m} e^{-t / \tau_{n}}, \quad \forall n, m \neq 0 .
$$

The center of mass diffusion constant $D$ follows from the time correlation function for the translational mode $n=0$,

$$
\begin{aligned}
D & =\lim _{t \rightarrow \infty} \frac{1}{6 L t}\left\langle\left[\boldsymbol{\chi}_{0}(t)-\chi_{0}(0)\right]^{2}\right\rangle \\
& =\frac{k_{B} T}{3 \pi \eta L}\left[1+\frac{\sqrt{6}}{\sqrt{\pi} L} \int_{d}^{L} \frac{L-s}{\sqrt{\sigma(s)}} \exp \left(-\frac{3 d^{2}}{2 \sigma(s)}\right) d s\right] .
\end{aligned}
$$

The FCS correlation function includes the dynamic structure factor, which is defined as [30] 


$$
S(\boldsymbol{q}, t)=\frac{1}{L^{2}} \int_{-L / 2}^{L / 2} \int_{-L / 2}^{L / 2}\left\langle\exp \left\{-i \boldsymbol{q}\left[\boldsymbol{r}(s, t)-\boldsymbol{r}\left(s^{\prime}, 0\right)\right]\right\}\right\rangle d s d s^{\prime}
$$

for the continuous chain, where $\boldsymbol{q}$ is the scattering vector. Since the distribution of distances is Gaussian in our model and by exploiting the correlations (28), the dynamic structure factor is given by the well-known expression [30]

$$
S(\boldsymbol{q}, t)=\frac{1}{L^{2}} \int_{-L / 2}^{L / 2} \int_{-L / 2}^{L / 2} \exp \left[-\frac{q^{2}}{6} \Phi_{s s^{\prime}}(t)\right] d s d s^{\prime},
$$

where [36]

$$
\begin{aligned}
\Phi_{s s^{\prime}}(t)= & \left\langle\left[\boldsymbol{r}(s, t)-\boldsymbol{r}\left(s^{\prime}, 0\right)\right]^{2}\right\rangle=6 D t+\sigma\left(s-s^{\prime}\right) \\
& +\frac{2 k_{B} T}{\pi \eta} \sum_{n=1}^{\infty} \tau_{n} \psi_{n}(s) \psi_{n}\left(s^{\prime}\right)\left(1-e^{-t / \tau_{n}}\right)
\end{aligned}
$$

and $\sigma$ is defined in Eq. (13).

\section{FCS correlation function}

As discussed in Sec. II A, the dynamic structure factor determines the FCS correlation function (3). Assuming a homogeneously labeled continuous semiflexible chain, the correlation function $g_{\mathrm{D}}(t)$ is easily calculated via Eqs. (3), (4), and (31). The final expression reads

$$
\begin{aligned}
g_{\mathrm{D}}(t)= & \bar{g} \int_{-L / 2}^{L / 2} \int_{-L / 2}^{L / 2}\left(1+\frac{2 \Phi_{s s^{\prime}}(t)}{3 r_{0}^{2}}\right)^{-1} \\
& \times\left(1+\frac{2 \Phi_{s s^{\prime}}(t)}{3 z_{0}^{2}}\right)^{-1 / 2} d s d s^{\prime}
\end{aligned}
$$

In an experiment, only a certain number of monomers $(N)$ are labeled. The structure factor of the continuous chain is then given by

$$
S(\boldsymbol{q}, t)=\frac{1}{N^{2}} \sum_{i=1}^{N} \sum_{j=1}^{N}\left\langle\exp \left\{-i \boldsymbol{q}\left[r_{i}(t)-r_{j}(0)\right]\right\}\right\rangle,
$$

with $r_{i}(t)=r\left(s_{i}, t\right)$ and $s_{i}$ the contour coordinate of the labeled monomer. The average is calculated with the distribution function of the continuous chain. The correlation function $g_{\mathrm{D}}(t)$ is then given by

$$
g_{\mathrm{D}}(t)=\hat{g} \sum_{i=1}^{N} \sum_{j=1}^{N}\left(1+\frac{2 \Phi_{i j}(t)}{3 r_{0}^{2}}\right)^{-1}\left(1+\frac{2 \Phi_{i j}(t)}{3 z_{0}^{2}}\right)^{-1 / 2},
$$

where $\Phi_{i j}=\left\langle\left[\boldsymbol{r}\left(s_{i}, t\right)-\boldsymbol{r}\left(s_{j}, 0\right)\right]^{2}\right\rangle$. The factors $\bar{g}$ and $\hat{g}$ comprise various constants, which will not be of any relevance for the further study, because we will focus on the normalized correlation function $G_{\mathrm{D}}(t)=g_{\mathrm{D}}(t) / g_{\mathrm{D}}(0)$ (cf Sec. II A).

Analytical approximations for the eigenfunctions and relaxation times can be derived in the limits of flexible chains $(p L \rightarrow \infty)$ and semiflexible chains $(p L<1)$, respectively. In the first case, the stretching modes dominate, whereas in the second case the bending modes dominate $[35,36]$. For $p L$ $\rightarrow \infty$, the relaxation times (27) reduce to

$$
\begin{gathered}
\tau_{n}=\frac{\eta}{\pi k_{B} T p}\left(\frac{L}{n}\right)^{2}, \\
\widetilde{\tau}_{n}=\frac{\eta}{\sqrt{3 \pi} k_{B} T}\left(\frac{L}{p n}\right)^{3 / 2},
\end{gathered}
$$

where the latter ones are the well-known Zimm relaxation times $[29,30]$. Hence, the relaxation times for a flexible polymer in solution are proportional to $L^{3 / 2}$ and vary with $n^{-3 / 2}$. In the limit $p L<1$, the relaxation times with mode numbers $n>1$ are approximately given by

$$
\begin{gathered}
\tau_{n}=\frac{64 \eta p L^{4}}{\pi^{3} k_{B} T(2 n-1)^{4}}, \\
\tilde{\tau}_{n} \approx \frac{64 \eta p L^{4}}{\sqrt{6 \pi^{5}} k_{B} T(2 n-1)^{4} \ln (\Lambda L / d)} .
\end{gathered}
$$

For $n=1$, the relaxation time

$$
\tau_{1}=\frac{\pi \eta L^{3}}{12 k_{B} T}
$$

is obtained in the limit $p L \rightarrow 0$ [35]. The dimensionless quantity $\Lambda L / d$ is a measure of the hydrodynamic aspect ratio of the macromolecule. Since semiflexible chains without hydrodynamic interactions already display the dependence $\tau_{n}$ $\sim L^{4} /(2 n-1)^{4} \quad[33,34,36,52]$, hydrodynamic interactions have only a small influence on the mode number and chain length dependence of the relaxation times (for more details, see Refs. [35,36]).

In order to elucidate the influence of bending and stretching modes on the FCS correlation function, we consider the limits where all internal modes are either stretching modes or bending modes. We like to point out that these limits are not strictly valued for the model discussed, but can serve as first approximations. Moreover, the analysis is only carried out for continuously labeled polymers.

Using the expression for the stretching relaxation times (37), the eigenfunctions in the limit $p L \gg 1$, and by converting the sum over $n$ in Eq. (32) to an integral $\left(t / \widetilde{\tau}_{1} \ll 1\right)$ [30], we find

$$
\begin{aligned}
\frac{2 \Phi_{s}(t)}{3 r_{0}^{2}}= & \frac{2|s|}{3 p r_{0}^{2}}+\frac{4 D t}{r_{0}^{2}}+\frac{8}{\pi}\left(\Gamma_{Z} t\right)^{2 / 3} \\
& \times \int_{0}^{\infty} \cos \left(\frac{s x}{6 p r_{0}^{2}}\left(\Gamma_{Z} t\right)^{-2 / 3}\right)\left[1-\exp \left(-\frac{x^{3 / 2}}{\sqrt{2}}\right)\right] \frac{d x}{x^{2}},
\end{aligned}
$$

with

$$
\Gamma_{Z}=\frac{k_{B} T}{6 \pi \eta r_{0}^{3}} .
$$

The decay rate $\Gamma_{Z}$ is identical to the decay rate of the dynamic structure factor [30]. But in FCS $1 / r_{0}$ plays the role of the scattering vector. Also in this situation, the decay rate is independent of chain length and persistence length. For 
$\Gamma_{Z} \gg 1$, the time dependence in the integral (41) can be neglected and we find the approximation

$$
\frac{2 \Phi_{s}(t)}{3 r_{0}^{2}}=\frac{2|s|}{3 p r_{0}^{2}}+\frac{4 D t}{r_{0}^{2}}+5.42\left(\Gamma_{Z} t\right)^{2 / 3}
$$

i.e., the well-known $t^{2 / 3}$ time dependence of the mean square displacement for flexible polymers is obtained $[29,30]$.

The correlation function is then given by

$$
g_{\mathrm{D}}^{f}(t)=2 \bar{g} L \int_{0}^{L}\left(1+\frac{2 \Phi_{s}(t)}{3 r_{0}^{2}}\right)^{-1}\left(1+\frac{2 \Phi_{s}(t)}{3 z_{0}^{2}}\right)^{-1 / 2} d s .
$$

(The applied approximations are valid in a certain time window. In the limit $t \rightarrow \infty$, a better approximation is obtained when the term $(1-s / L)$ is included in the integral over $s$. In this limit the intramolecular dynamics is negligible.)

The integral can be evaluated analytically, which yields

$$
\begin{aligned}
g_{\mathrm{D}}^{f}(t)= & \frac{6 \bar{g} p r_{0}^{2} L f}{\sqrt{f^{2}-1}}\left[\operatorname{acoth}\left(\frac{f^{2}+\frac{4 D t}{r_{0}^{2}}+5.42\left(\Gamma_{Z} t\right)^{2 / 3}}{f^{2}-1}\right)^{1 / 2}\right] \\
& -\operatorname{acoth}\left[\left(\frac{f^{2}+\frac{2 L}{3 p r_{0}^{2}}+\frac{4 D t}{r_{0}^{2}}+5.42\left(\Gamma_{Z} t\right)^{2 / 3}}{f^{2}-1}\right)^{1 / 2}\right],
\end{aligned}
$$

where $f=z_{0} / r_{0}\left[p L \gg 1, \Gamma_{Z} t \gg 1(42)\right]$.

Assuming that all relaxation times are bending relaxation times (39), $\Phi_{s s^{\prime}}(t)$ becomes $\left(s-s^{\prime} \rightarrow s\right)$

$$
\begin{aligned}
\frac{2 \Phi_{s}(t)}{3 r_{0}^{2}}= & \frac{2 s^{2}}{3 r_{0}^{2}}+\frac{4 D t}{r_{0}^{2}}+\frac{2^{3 / 2} 3^{3 / 4}}{\pi}\left(\Gamma_{B} t\right)^{3 / 4} \\
& \times \int_{0}^{\infty} \cos \left(\frac{\pi s x}{2 L}\left(\Gamma_{c} t\right)^{-1 / 4}\right)\left[1-\exp \left(-x^{4}\right)\right] \frac{d x}{x^{4}},
\end{aligned}
$$

with

$$
\begin{gathered}
\Gamma_{B}=\frac{\sqrt{6} p^{1 / 3} k_{B} T \ln (\Lambda L / d)}{3 \pi^{3 / 2} \eta r_{0}^{8 / 3}}, \\
\Gamma_{c}=\frac{\sqrt{6} \pi^{5 / 2} k_{B} T \ln (\Lambda L / d)}{64 \eta p L^{4}} .
\end{gathered}
$$

For $\Gamma_{c} t \gg 1$, Eq. (46) reduces to

$$
\frac{2 \Phi_{s}(t)}{3 r_{0}^{2}}=\frac{2 s^{2}}{3 r_{0}^{2}}+\frac{4 D t}{r_{0}^{2}}+1.65\left(\Gamma_{B} t\right)^{3 / 4} .
$$

The time dependence $t^{3 / 4}$ of $\Phi_{s}(t)$ applies to semiflexible polymers in solution as well as in melt $[33-36,53,54]$. The correlation function again follows from Eq. (44). Evaluation of the integral yields

$$
\begin{aligned}
g_{\mathrm{D}}^{b}(t)= & 2 \bar{g} L f \sqrt{\frac{3 r_{0}^{2}}{2\left(f^{2}-1\right)}}\left(1+\frac{4 D t}{r_{0}^{2}}+1.65\left(\Gamma_{B} t\right)^{3 / 4}\right)^{-1 / 2} \\
& \times \arctan \left\{\sqrt { \frac { 2 ( f ^ { 2 } - 1 ) L ^ { 2 } } { 3 r _ { 0 } ^ { 2 } } } \left[\left(1+\frac{4 D t}{r_{0}^{2}}+1.65\left(\Gamma_{B} t\right)^{3 / 4}\right)\right.\right. \\
& \left.\left.\times\left(f^{2}+\frac{2 L^{2}}{3 r_{0}^{2}}+\frac{4 D t}{r_{0}^{2}}+1.65\left(\Gamma_{B} t\right)^{3 / 4}\right)\right]^{-1 / 2}\right\}
\end{aligned}
$$

$\left[p L \ll 1, \Gamma_{c} t \gg 1(48)\right]$. In Sec. IV, we will discuss the dependence of the FCS correlation function on the persistence length and compare the theoretical results with experimental measurements.

\section{EXPERIMENTAL SETUP}

We used a commercial FCS setup by Carl Zeiss (Jena, Germany) consisting of the module ConfoCor2 [55] and the microscope Axiovert200 with a Zeiss C-Apochromat $40 \times$ NA 1.2 water immersion objective. For excitation the $488 \mathrm{~nm}$ line of an argon ion laser was used. The laser intensity for all measurements was $60 \mu \mathrm{W}$ in sample space. The emitted light was split up by a 50/50 mirror and fed to two identical independent detection channels with $505 \mathrm{~nm}$ long pass filters and pinholes with $70 \mu \mathrm{m}$ diam. The intensities coming from the two avalanche photodiodes were cross correlated. Artifacts such as detector afterpulsing or other detection noise do not occur in the cross-correlation function.

Calibration measurements were done with a $10 \mathrm{nM}$ rhodamine $6 \mathrm{G}$ solution. For all measurements $r_{0}$ was between 180 and $200 \mathrm{~nm}$. The ratio $z_{0} / r_{0}$ was always between 7 and 8 .

$\lambda$-phage DNA (48 502 bp) was purchased from MBI Fermentas (St. Leon-Rot, Germany). The linear 7250 and $2686 \mathrm{bp}$ DNA were the result of cutting the plasmids M13mp18 (MBI Fermentas) and pUC18 (MBI Fermentas) with the restriction enzyme ECO R1 (New England BioLabs, Beverly, MA, USA).

All DNA samples were kept in $10 \mathrm{mM}$ Tris ( $\mathrm{pH}$ 8.0), $1 \mathrm{mM}$ EDTA, and $100 \mathrm{mM} \mathrm{NaCl}$. DNA pieces were labeled with the intercalating fluorescent dye YOYO-1 (absorption: $491 \mathrm{~nm}$, emission: $509 \mathrm{~nm}$ ) (Molecular Probes, Eugene, OR, USA, Y-3601). The number of dyes $N$ per chain was tuned by adding a dye solution with an appropriate concentration. The mixture was gently shaken at $37^{\circ} \mathrm{C}$ to have a preferably homogeneous dye distribution along the chain. Nevertheless, the number of dyes per chain and their distance are statistically distributed and can have large fluctuations especially at low label densities.

Monomeric G-actin from rabbit muscle $\left(M_{\mathrm{w}}=42 \mathrm{kDa}\right)$ was a kind gift from the group of M. Bärmann (Physics Department E22, Technical University München, Germany). G-actin was fluorescently labeled by attaching a rhodamine dye covalently to the protein.

Unlabeled and labeled G-actin were mixed in G-buffer (2 mM Tris/HCl pH 7.4, $0.5 \mathrm{mM}$ ATP, $0.2 \mathrm{mM} \mathrm{CaCl}_{2}$, $0.2 \mathrm{mM}$ Dithiothreitol, and $0.2 \% \mathrm{NaN}_{3}$ ) at a ratio of $10: 1$, and then polymerized by changing to F-buffer conditions (Gbuffer $+2 \mathrm{mM} \mathrm{MgCl}_{2}$ and $100 \mathrm{mM} \mathrm{KCl}$ ). The total G-actin 
concentration during the polymerization was $2 \mathrm{mg} / \mathrm{ml}$. Gelsolin was added to the solution to control the length of the filaments. This capping protein inhibits polymerization. The molar ratio of G-actin and gelsolin determines the average length $\langle L\rangle$

$$
\langle L\rangle=\frac{[\mathrm{G}-\text { actin }] /[\text { gelsolin }]}{370} \mu \mathrm{m}
$$

After 20 min, phalloidin (Molecular Probes, P-3457) (phalloidin: G-actin=1:4) was added to stabilize the filaments. These filaments could now be diluted without depolymerization. The average label distance along the chains was $\approx 28 \mathrm{~nm}$ as every tenth monomer was labeled.

\section{RESULTS AND DISCUSSION}

As is well known, the decay of the dynamic structure factor is given by $S(\boldsymbol{q}, t)=\exp \left(-\boldsymbol{q}^{2} D t\right)$ for small scattering vectors $q\left\langle\boldsymbol{r}_{g}^{2}\right\rangle^{1 / 2} \ll 1[56,57]$. Similarly, the diffusive decay of the FCS correlation function is determined by the diffusion coefficient for $\left\langle\boldsymbol{r}_{g}^{2}\right\rangle^{1 / 2} \ll r_{0}$ and only the overall translational motion of a macromolecule is observed. In order to study the intramolecular dynamics, typical chain dimensions as the radius of gyration should obey the relation $\left\langle\boldsymbol{r}_{g}^{2}\right\rangle^{1 / 2} \gg r_{0}$. For flexible polymers, the persistence length should be much smaller than $r_{0}$, i.e., $p r_{0} \gg 1$. Combining the two conditions for flexible polymers (in $\theta$ solvent) leads to the inequalities $r_{0} / L \gg 1 / p L \gg\left(r_{0} / L\right)^{2}$. To satisfy this relation, rather long polymers are required. For an FCS microscope with $r_{0}$ $\approx 200 \mathrm{~nm}$, a reasonable polymer length would be $p L \approx 10^{4}$. Even long DNA molecules are smaller than $p L=10^{3}$; thus, no strict flexible chain behavior is obtained.

To study the intramolecular dynamics of molecules by FCS, the experimentally accessible time scale has to be shorter than the longest relaxation time $\widetilde{\tau}_{1}[(37)$, (39), and (40)] of the polymer. For times larger than the longest relaxation time, the intramolecular contribution to $\Phi_{s s^{\prime}}(t)$ (32) is time independent and the center-of-mass diffusion term determines the FCS correlation function. Hence, the intramolecular dynamics yields a significant contribution to $\Phi_{s s^{\prime}}(t)$ for $t \ll \widetilde{\tau}_{1}$ only.

The diffusive part of the FCS function for a continuously labeled polymer is calculated using Eq. (33) as well as (35) with $\Phi_{s s^{\prime}}(t)$ defined in Eq. (32). By changing the number of labels $(N)$, we ensure that the results of the two expressions coincide. The wave numbers and the relaxation times $\tau_{n}$ are calculated from the nonlinear equations following from the boundary conditions (17) and (18), [35-38]. To avoid artifacts by truncating the sum over the mode numbers in Eq. (32), we choose a sufficiently large maximum mode number and ensure that the curves which we will present in the following correspond to the limit of an infinite sum.

\section{A. FCS correlation function for flexible polymers}

In this section, we will discuss the diffusive part of the FCS correlation for polymers that approximately resemble flexible chains. In particular, we will address the dependence

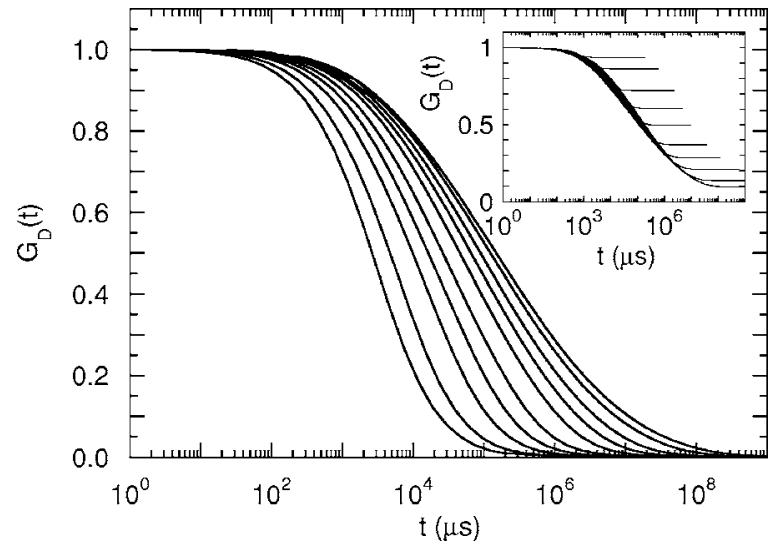

FIG. 1. Diffusive parts of the FCS correlation functions for continuously labeled (flexible) polymers of various lengths: $p L=10^{1}$, $2 \times 10^{1}, 5 \times 10^{1}, 10^{2}, 2 \times 10^{2}, 5 \times 10^{2}, 10^{3}, 2 \times 10^{3}, 5 \times 10^{3}$, and $10^{4}$ (left to right). Other parameters are $l_{p}=50 \mathrm{~nm}, d=2.5 \mathrm{~nm}, T$ $=300 \mathrm{~K}, \eta=0.01 \mathrm{P}, r_{0}=0.18 \mu \mathrm{m}$, and $f=7$. The inset shows the FCS correlation functions calculated with intramolecular contributions only ( $p L$ increases from top to bottom).

of the correlation function on the length of the polymer and the density of fluorescence labels. In order to compare the theoretical results with experimental measurements, we chose model parameters typical for DNA molecules. In particular, we use the persistence length $l_{p}=1 / 2 p=50 \mathrm{~nm}$, the thickness $d=2.5 \mathrm{~nm}$, temperature $T=300 \mathrm{~K}$, viscosity $\eta$ $=0.01 \mathrm{P}, r_{0}=0.18 \mu \mathrm{m}$, and $f=7$.

\section{Chain length dependence}

A variation of the chain length at fixed persistence length corresponds to a change from rather rigid $(p L \gtrsim 1)$ to flexible polymers $(p L \gg 1)$. (Note, $\Phi_{s s^{\prime}}$ not only depends on $p L$ but also on the ratios $r_{0} / L$ and $d / L$, which decrease with increasing chain length.) Hence, generally both stretching as well as bending modes contribute to $\Phi_{s s^{\prime}}$.

Figure 1 displays FCS correlation functions for continuously labeled chains of various lengths. The chain lengths increase from $p L=10$ (left) to $p L=10^{4}$ (right). The inset shows the intramolecular contributions only, i.e., $G_{\mathrm{D}}(t)$ corresponds to the FSC correlation function in the center-ofmass reference frame of a single polymer. With increasing chain length, the corresponding curves shift to longer times, the time window over which the correlation function decays increases, and the correlation functions seem to approach a limiting curve. The intramolecular contribution to the overall decay of the correlation function increases with increasing chain length (see inset of Fig. 1).

Alternatively, we can consider the function $1 / G_{\mathrm{D}}(t)-1$, which amplifies the time dependence of $G_{\mathrm{D}}(t)$ close to unity. If the correlation function displays a time dependence as assumed in Eq. (6), a double logarithmic plot will directly yield the exponent $\beta$ for small times.

Figure 2 shows correlation functions for various chain lengths in such a representation. For small $p L$ values, $1 / G_{\mathrm{D}}(t)-1$ increases essentially linearly with time (see also Fig. 3). Considering Eq. (5), we see that in the short time 


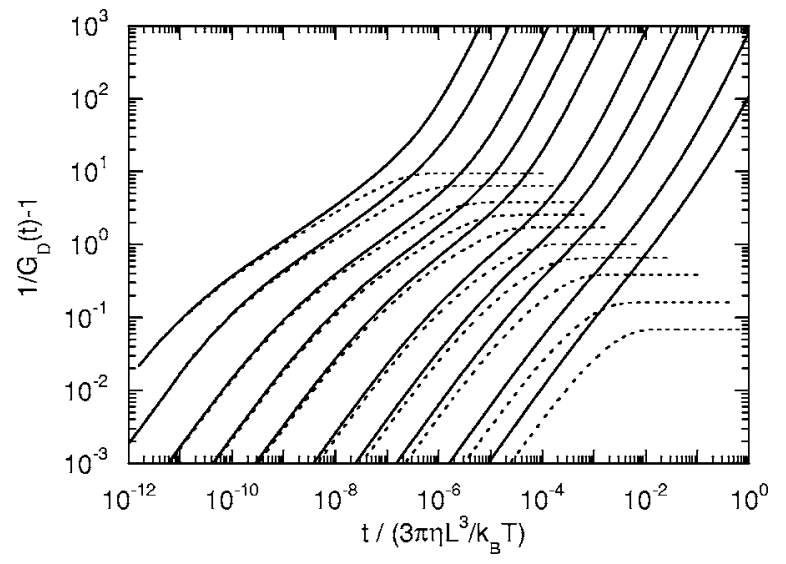

FIG. 2. FCS correlation functions for (flexible) polymers of various lengths ( $p L$ values see Fig. 1). The dashed lines are calculated taking into account intramolecular contributions only ( $p L$ increases from right to left).

regime $4 D t / r_{0}^{2}<1$, the decay of the correlation function is determined by the $x y$ component of the observation volume, which corresponds to the observed linear time dependence. For $4 D t / r_{0}^{2} / f^{2}>1$, all spatial components are important and $1 / G_{\mathrm{D}}(t)-1$ increases like $t^{3 / 2}$.

The dashed lines represent the intramolecular contributions to the correlation functions, which saturate at $t>\widetilde{\tau}_{1}$. This contribution to the correlation function is negligible for small $p L$ values. Hence, we do not discuss correlation functions for $p L<10$ at this point. With increasing chain length, the intramolecular contribution increases, which leads to reduced slopes of the curves for $t<\widetilde{\tau}_{1}$.

The time dependence of the slopes of $\log \left[1 / G_{\mathrm{D}}-1\right]$ is depicted in Fig. 3 for the various chain lengths. As already pointed out, for $p L=10$ and $t<0.1 \mathrm{~s}$, the slope is essentially equal to unity because diffusion dominates over the intramolecular dynamics [cf. Eq. (32)]. At sufficiently large times, all the slopes assume the value $\frac{3}{2}$, whereas for the intramolecular contribution a zero slope is found. With increasing polymer length, we find a time window with slopes significantly smaller than unity. This time window covers the ex-

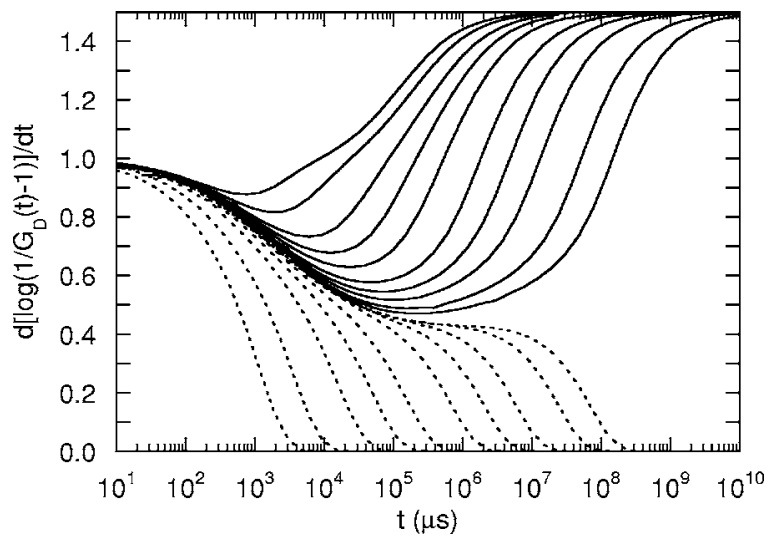

FIG. 3. Slopes of the functions $\log \left[1 / G_{\mathrm{D}}(t)-1\right]$ for various polymer lengths ( $p L$ values as in Fig. 1). The solid lines correspond to the total correlation function and the dotted lines to the intramolecular contribution ( $p L$ increases from left to right). perimentally accessible time scale. [The center of the experimental time window can be located by setting $G_{\mathrm{D}}\left(t_{c}\right) \approx 0.5$.] For $p L \approx 200$, the slope is on the order of $\frac{2}{3}$, the value predicted by Zimm theory [30]. For longer chains, we find a slope smaller than $\frac{1}{2}$ and the analytical expression (45) even predicts the time dependence $t^{1 / 3}$ for $\Gamma_{Z} t \gg 1, \Gamma_{Z} t \gg 4 D t / r_{0}^{2}$, a limiting value that is reached for a very flexible polymer. This is not surprising, since $1 / G_{\mathrm{D}}(t)-1$ does not yield the monomer mean square displacement, in general, and, hence, does not have to exhibit Zimm behavior. (Only for a single label follows the mean square displacement, cf. Sec. IV A 2.) It is rather a weighted function of the mean square displacements of all monomers [cf. Eqs. (41) and (44)]. This underlines our point that an adequate model is necessary to extract the physical properties of the polymer under investigation.

The various plots indicate that the intramolecular dynamics determines the decay of the FCS correlation function for sufficiently long chains $(p L \gg 10)$ and short enough times $\left(t \ll \tilde{\tau}_{1}\right)$. In this parameter range, the intramolecular dynamics is measured directly. This is reflected in Fig. 3. For long polymers, the derivative of $\log \left[1 / G_{\mathrm{D}}(t)-1\right]$, where $G_{\mathrm{D}}(t)$ is calculated taking only intramolecular contributions into account, develops a plateau, which extends over several decades. The slope calculated with the total correlation function assumes slightly larger values. The dominance of the intramolecular dynamics at large chain lengths is a consequence of the following facts: (i) $\widetilde{\tau}_{1} \sim L^{3 / 2}$, i.e., the relaxation time $\widetilde{\tau}_{1}$ increases with the chain length; (ii) $D \sim L^{-1 / 2}$, i.e., the diffusion constant decreases with the chain length; and (iii) the relaxation time $\Gamma_{Z}$ is independent of chain and persistence length. As far as the current parameters are concerned, the universal regime is reached for $p L \gtrsim 5000$. The time window of this regime increases with increasing polymer length.

In Fig. $4,1 / G_{\mathrm{D}}(t)-1$ of the numerically determined correlation function is compared to the analytical expression (45) for flexible polymers. For $p L>1000$, the full theoretical result is very well described by the analytical approximation over several decades. With decreasing chain lengths, the analytical approximation deviates more and more from the exact data. This is to be expected, since the approximation should apply for very flexible polymers. To achieve agreement, however, the time scale of the analytical expression is multiplied by 1.7. This is a consequence of the approximations involved in the calculation of the sum over modes in Eqs. (41) and (43). As numerical studies show, the major reason is that the cosine of Eq. (41) is replaced by one. Similarly, the correlation function of the intramolecular part is very well approximated by the analytical expression. Again the time has to be multiplied by a factor of 1.9. Although there is a quantitative disagreement between the time scales by approximately a factor of 2 the analytical correlation function captures the time dependence of the exact one.

In the limit of very short times, the theoretical correlation function predicts a dependence $g_{\mathrm{D}}^{f} \sim\left[1+2 D t / r_{0}^{2} f^{2}\right.$ $\left.+2.71\left(\Gamma_{z} t\right)^{2 / 3} / f^{2}\right]^{-1}$, i.e., we should observe an exponent of $\frac{2}{3}$ in the $1 / G_{\mathrm{D}}(t)-1$ representation. The numerical calculation, however, yields larger exponents for $t / \widetilde{\tau}_{1} \ll 1$, which approach unity for very small times. On the one hand, this is a consequence of the semiflexibility of a DNA molecule. For 


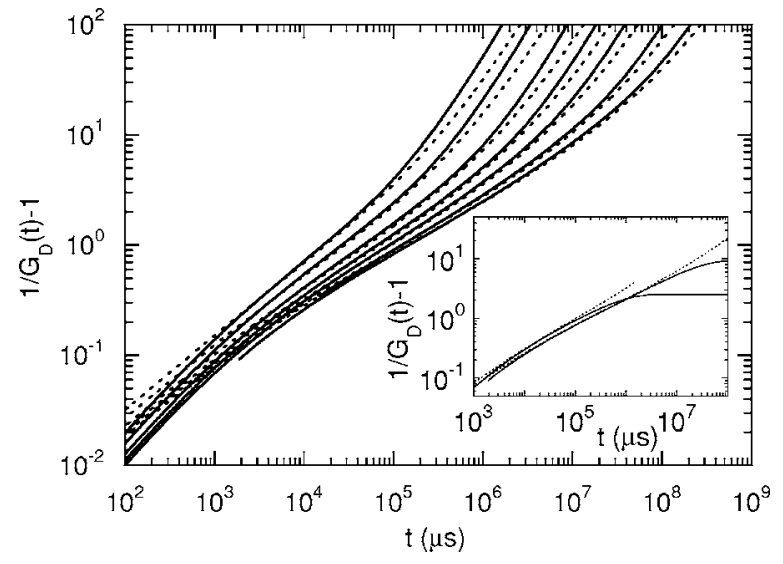

FIG. 4. Comparison of the full numerically determined FCS correlation function (solid lines) with the analytical approximation of Eq. (45) (dotted lines) for the polymer lengths $p L=10^{2}, 2 \times 10^{2}$, $5 \times 10^{2}, 10^{3}, 2 \times 10^{3}, 5 \times 10^{3}$, and $10^{4}$. (For the other parameters, see Fig. 1.) The time scale of the analytical solution is multiplied by 1.7 to match the time scale of the full numerical correlation function. Inset: Correlation functions taking into account intramolecular contributions only for $p L=10^{3}$ and $10^{4}$ (solid lines: theoretical expression, dotted lines: analytical approximation). The time scale of the analytical solution is multiplied by 1.9 ( $p L$ increases from left to right).

short times bending modes determine the relaxation behavior rather than stretching modes. On the other hand, for $\Gamma_{Z} t \ll 1$, the time dependence of the cosine function in Eq. (41) cannot be neglected anymore because the function rapidly oscillates. As is clear from numerical calculations using the full expression (32), this gives rise to a linear time dependence. To observe stretching modes only, much more flexible chains with a much shorter persistence length have to be considered.

The dependence of the FCS correlation function on chain length can be studied by measuring DNA fragments produced in the way described in Sec. III. The FCS correlation functions for three DNA lengths are presented in Fig. 5. The numbers of labels are sufficiently large to correspond to continuously labeled chains. The theoretical curves agree very well with the experimental data. As far as the parameters are concerned, we set $r_{0}=0.2 \mu \mathrm{m}$ and $f=7$. As indicated by the dashed lines, the contribution of the intramolecular dynamics to the FCS signal is small for all three chain lengths. Only for $\lambda$-phage DNA does it provide an appreciable contribution (see Fig. 6). This is also reflected in the slope of $\log \left[1 / G_{\mathrm{D}}(t)-1\right]$ shown in Fig. 6. For the shortest DNA, the slope is essentially equal to unity, whereas a slightly smaller value is found for the $7250 \mathrm{bp}$ DNA molecules. For $\lambda$-phage DNA a slope of $\approx \frac{3}{4}$ can be inferred. This is the value presented in Ref. [16]. However, the slope does not correspond to the value of a semiflexible chain, but is rather a consequence of only a partial contribution of the intramolecular dynamics to the total FCS correlation function.

Strictly speaking, there is no fit parameter in the theoretical model. Indeed, the experimental time scales for the two shorter DNA fragments agree with the time scale of the model within $\sim 10 \%$. For these molecules, we find the diffu-

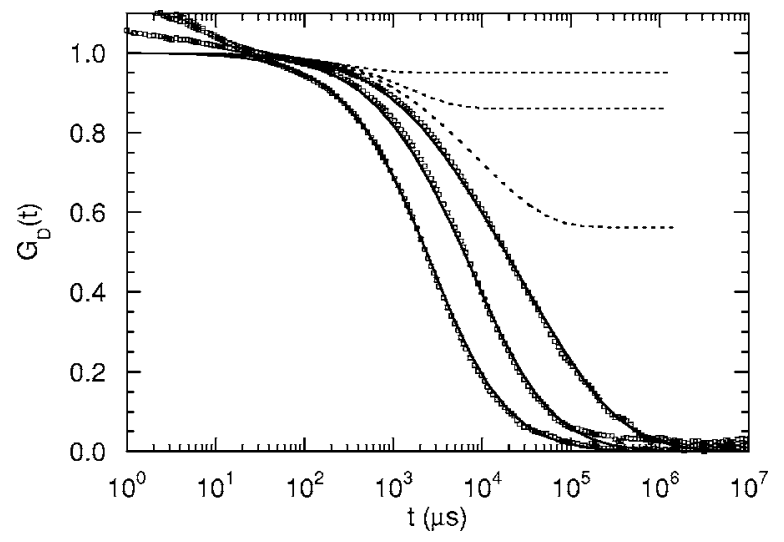

FIG. 5. Comparison of theoretical FCS correlation functions (solid lines) with experimental measurements (squares) on densely labeled DNA for $p L=9$ (2686 bp), 25 (7250 bp), and 165 (48 $502 \mathrm{bp)} \mathrm{(left} \mathrm{to} \mathrm{right).} \mathrm{The} \mathrm{dotted} \mathrm{lines} \mathrm{represent} \mathrm{intramolecular}$ contributions only ( $p L$ increases from top to bottom).

sion constants $D \approx 4.3 \mu \mathrm{m}^{2} / \mathrm{s}$ (2686 bp DNA) and $D$ $\approx 2.3 \mu \mathrm{m}^{2} / \mathrm{s}$ (7250 bp DNA), respectively. For $\lambda$-phage DNA, however, we obtain the diffusion coefficient $D$ $\approx 1.1 \mu \mathrm{m}^{2} / \mathrm{s}$, in agreement with results presented in Ref. [16]. This value is approximately a factor of two larger than the value extracted from dynamic light-scattering experiments [58]. The origin of the discrepancy remains to be clarified.

\section{Variation of label density}

The FCS correlation function depends not only on chain length but also on the density of labels along the polymer contour [16]. Thus far, all theoretical curves correspond to a homogeneous and continuous distribution of labels. The correlation function for a discrete distribution of labels follows from Eq. (35). As before, we assume an underlying continuous chain, but only a discrete number of labels yield a fluo-

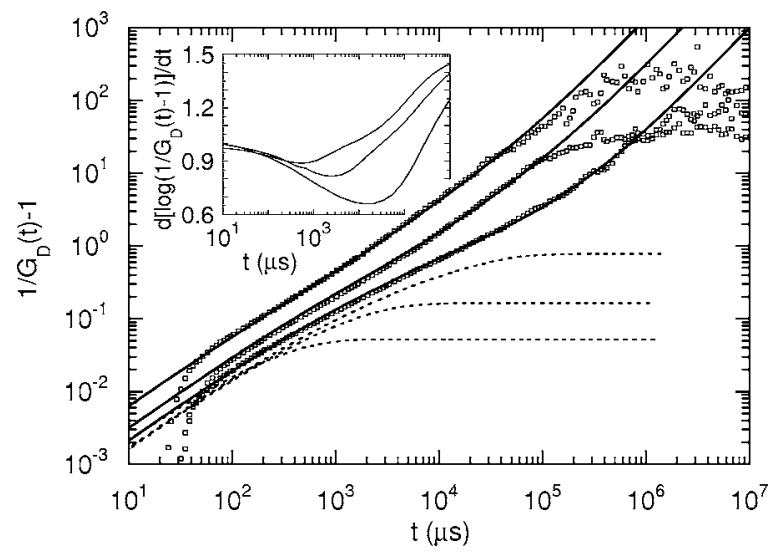

FIG. 6. Comparison of the theoretical FCS correlation function (solid lines) with experimental measurements on DNA (squares) for $p L=9,25$, and 165 (left to right). The dotted lines represent intramolecular contributions only ( $p L$ increases from bottom to top). Insert: Slopes of the theoretical correlation functions $\log \left[1 / G_{\mathrm{D}}(t)\right.$ $-1]$. 


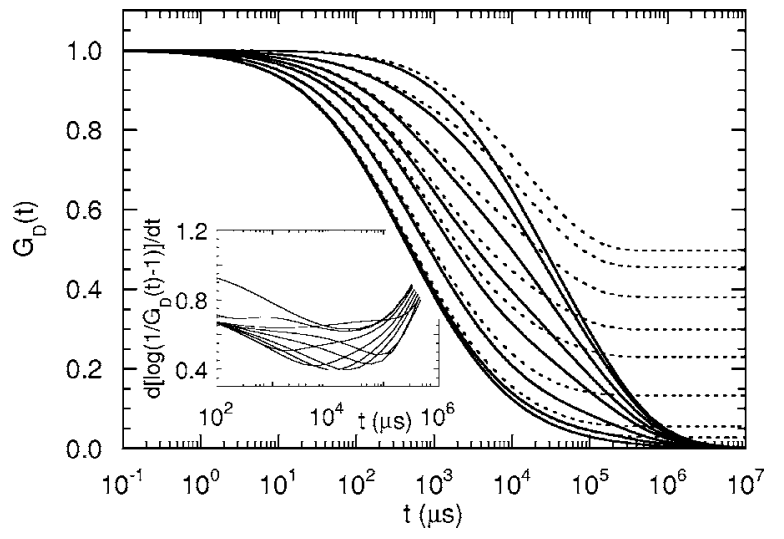

FIG. 7. FCS correlation functions for various label densities: $N=1,2,3,5,7,11,21$, continuum (left to right). The dotted lines give the intramolecular contributions only. The chain length is $p L$ $=200$. The other parameters are the same as in Fig. 1. Inset: Slopes of $\log \left(1 / G_{\mathrm{D}}-1\right)$ for various label densities (increasing from right to left). The slopes for $N=51$ are presented in addition to those given above.

rescence signal. For simplicity, we assume that the distances between neighboring labels are constant. A random distribution would also be possible, but then we would have to average over the distribution of the labels.

A limiting situation is a single label on the DNA molecule. The dynamic structure factor (34) is then identical to the incoherent dynamic structure factor and $\Phi_{s s^{\prime}}(32)$ reduces to the mean square displacement

$$
\Phi_{s s}(t)=6 D t+\frac{2 k_{B} T}{\pi \eta} \sum_{n=1}^{\infty} \tau_{n} \psi_{n}^{2}(s)\left[1-\exp \left(-t / \widetilde{\tau}_{n}\right)\right]
$$

The correlation function itself reads

$$
g_{\mathrm{D}}(s, t)=\left(1+\frac{2 \Phi_{s s}(t)}{3 r_{0}^{2}}\right)^{-1}\left(1+\frac{2 \Phi_{s s}(t)}{3 z_{0}^{2}}\right)^{-1 / 2},
$$

with $s$ the position of the label along the chain contour. Thus, the correlation function depends on the position of the label. However, it is a quantitative rather than a qualitative difference. Experimentally, single labeled DNA molecules have been measured in Ref. [59].

By inversion, the mean square displacement is obtained from $g_{\mathrm{D}}(s, t)(53)$. We can use this relation to test how closely $1 / G_{\mathrm{D}}(t)-1$ resembles the mean square displacement. The numerical calculation shows that $1 / G_{\mathrm{D}}(t)-1$ agrees very well with the mean square displacement up to the time where $4 D t / r_{0}^{2}>1$, i.e., up to the transition to free diffusion of the whole molecule. At even larger times, the mean square displacement is $\sim t$, whereas $1 / G_{\mathrm{D}}(t)-1 \sim t^{3 / 2}$.

Figure 7 depicts FCS correlation functions for various label densities. The chain length is $p L=200$ and the other parameters are identical to those of the previous subsection. For $N=1$, one of the chain ends is labeled and for $N=2$ both ends are labeled. For $N \geqslant 3$, only curves for an odd number of labels are presented, where the central point of the poly- mer is always labeled. The shape of the curves changes significantly with increasing label number. As discussed above, for a single label only the incoherent self-term (52) contributes to the decay of the correlation function. The correlation function for a molecule with both end points labeled is at short times identical to that of a single labeled polymer. In this time regime, the individual incoherent terms of the ends contribute to the decay of the correlation function. However, there is also a coherent term $\Phi_{-L / 2 L / 2}(t)$. At short times its contribution to the correlation function is too small to be detected; the function $\sigma(L)$ [cf. Eqs. (32) and (35)] is much larger than the sum over the eigenfunctions of Eq. (32). With increasing time, however, the latter sum is comparable to the sum in the self-term (52) and modifies the decay of the correlation function as is visible in Fig. 7 for $t \gtrsim 10^{3} \mu \mathrm{s}$. Labeling of further points leads to a shift of the correlation function curves to larger times. This is, on the one hand, related to the appearance of more and more coherent terms with increasing label density. On the other hand, the chain ends are more mobile than the central part of the polymer, i.e., the mean square displacement of a chain end is larger than the mean square displacement of the chain center in the time window of the decay of the correlation function. This leads to a shift of the whole curve to larger times even if only incoherent terms are included in the calculation of $g_{\mathrm{D}}(s, t)$. Naturally, there is a limiting curve for continuously labeled polymers.

The various contributions to the decay of the correlation function affect its shape. For a single label, only its mean square displacement enters the correlation function. Thus, the slopes of $\log \left[1 / G_{\mathrm{D}}-1\right]$ are close to $\frac{2}{3}$ over a broad time window (see inset of Fig. 7). The appearing coherent term in $\Phi_{i j}$ for larger label densities leads to a decrease of the intramolecular contribution to the correlation function as seen by the dashed lines of Fig. 7. The slopes of $\log \left[1 / G_{\mathrm{D}}(t)\right.$ -1] then become significantly smaller than the value for a single labeled polymer in a certain time window. In this time regime, a superposition of the intramolecular dynamics and the overall diffusion of a molecule is observed. Thus, one has to be carefully relating the observed exponent to the intramolecular dynamics. At larger label densities, the relative contribution of the center-of-mass diffusion compared to the intramolecular dynamics increases and the slopes assume larger values. Again, the correlation function is determined by the center-of-mass motion rather than by the intramolecular motion alone. For label numbers $N \gtrsim 50$, the correlation functions are hardly distinguishable from the limiting function for a continuously labeled chain.

\section{B. FCS correlation function for semiflexible polymers}

The dynamics of DNA molecules discussed in the last section is mainly determined by stretching modes, although the bending modes yield an appreciable contribution to the dynamics particularly at short chain lengths. Thus, DNA molecules are semiflexible polymers if they are sufficiently short. In this section we focus on actin filaments, which are much more rigid than DNA molecules, and hence, bending modes dominate the relaxation behavior. To describe the ac- 


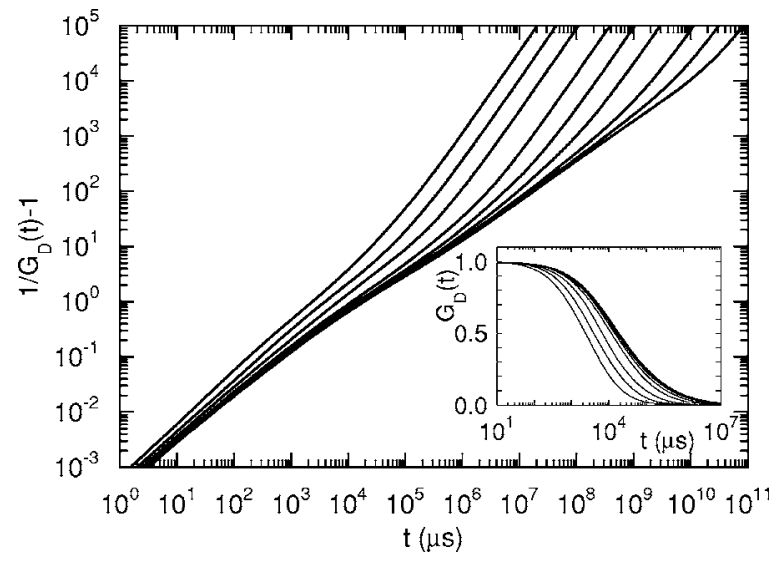

FIG. 8. FCS correlation functions for (rigid) polymers of various length: $p L=0.0085,0.017,0.035,0.085,0.17,0.35,0.85$, $1.7,3.5$ (left to right). Further parameters are: $l_{p}=16 \mu \mathrm{m}$ and $d$ $=8 \mathrm{~nm}$. The remaining parameters are the same as in Fig. 1. The inset shows the FCS correlation functions in a semilogarithmic representation.

tin filaments, we use the parameters $l_{p}=16 \mu \mathrm{m}[36,60]$ and $d=8 \mathrm{~nm}$. The other parameters are the same as for the DNA molecules (see Sec. IV A). We would like to point out that the persistence length of an actin filament is approximately two orders of magnitude larger than the focus $r_{0}$.

To study the dependence of the FCS correlation function on the length of an actin filament, we systematically vary its length from approximately $100 \mathrm{~nm}$ to $100 \mu \mathrm{m}$. These lengths correspond to the range $10^{-3}<p L<5$, i.e, all of them are rather rigid. Finally, we use the theoretical expression following from the preaveraging approximation (cf. Ref. [36]) to determine $\Lambda L / d$.

Figure 8 displays $G_{\mathrm{D}}(t)$ for actin-filament-like semiflexible polymers of various lengths. The chain length increases from left to right. The various curves exhibit a decrease of the slope with increasing chain length $\left(t<\widetilde{\tau}_{1}\right)$ and seem to approach a limiting curve. We will address the latter point further on down. As shown in Fig. 9, the overall diffusion of the polymer determines the correlation function for $p L$ $\lesssim 0.02$. The intramolecular contribution is small. For large $p L$ values, however, the intramolecular dynamics dominates more and more the time dependence in the experimentally accessible time window $10^{-2}<1 / G_{\mathrm{D}}(t)-1<10^{2}$. In fact, the correlation function is completely determined by the intramolecular dynamics at large $p L$ values. Hence, for such lengths the intramolecular dynamics is measured directly. The reason for this is the strong dependence of the $\tau_{n} \mathrm{~s}$ (38) on chain length. In particular, the longest relaxation time shifts very fast to large times and extends the time range in which the intramolecular dynamics dominates over the whole experimentally accessible time window.

The dominance of the intramolecular dynamics is also reflected in the slopes of the functions $\log \left[1 / G_{\mathrm{D}}(t)-1\right]$, which are displayed in Fig. 10. For $p L>0.17$ and taking into account the intramolecular contributions only, the slopes are close to those of the overall correlation functions. As expected, the slopes for very short polymers are of the order of unity at short times and turn over to $\frac{3}{2}$ at large times. With

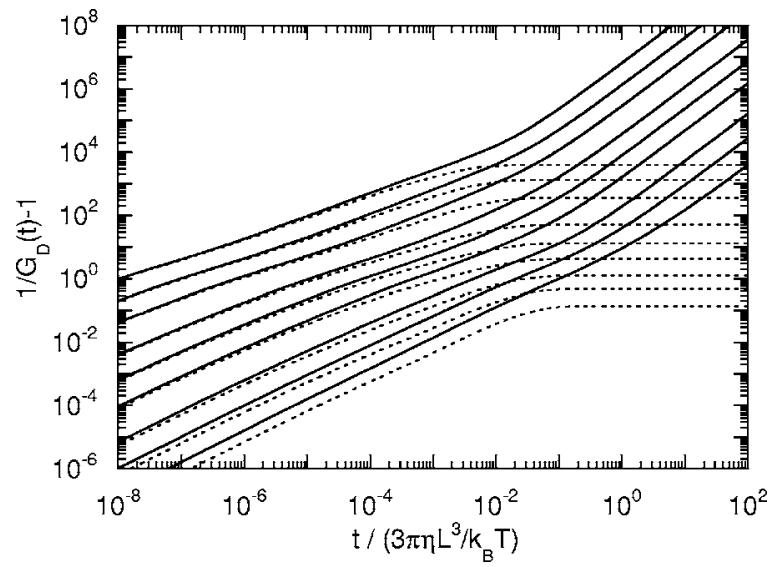

FIG. 9. FCS correlation functions for (rigid) polymers of various lengths (increasing from right to left). Solid lines: total correlation functions; dotted lines: intramolecular contributions. The parameters are the same as in Fig. 8.

increasing chain length two other regimes develop. Before the diffusive time regime $(t \rightarrow \infty)$ begins, a plateau appears with a slope close to $\frac{3}{4}$, corresponding to the dynamics of semiflexible polymers. A regime with slopes on the order of 0.65 is present at somewhat shorter times. The latter regime is within the experimental time window. The increase of the slope for $t \rightarrow 0$ has been explained in Sec. IV A.

The reason for the unexpected time dependence of the correlation function with an exponent of $\approx \frac{2}{3}$ can be understood by the analytical approximations presented in Sec. II D. As shown by Fig. 11, the time correlation functions are very well described by the analytical expression (50). Only the time scale has to be adjusted to a certain extent. The main reason for this adjustment is that the cosine term of Eq. (46) is replaced by unity in the analytical approximation.

For the appropriate limits, $G_{\mathrm{D}}(t)$ is well approximated by

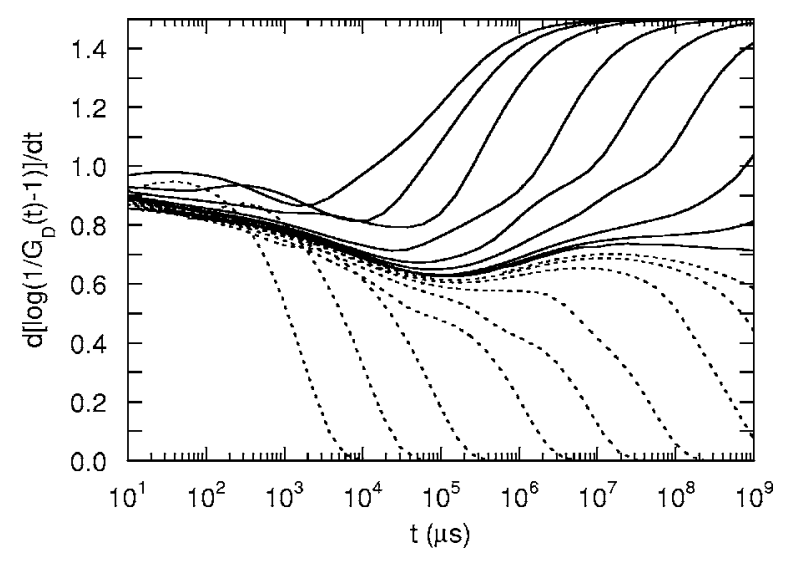

FIG. 10. Slopes of the function $\log \left[1 / G_{\mathrm{D}}(t)-1\right]$ for various polymer lengths (increasing from left to right; $p L$ values as in Fig. $8)$. The solid lines correspond to the total correlation function and the dotted lines to the intramolecular contributions only. 


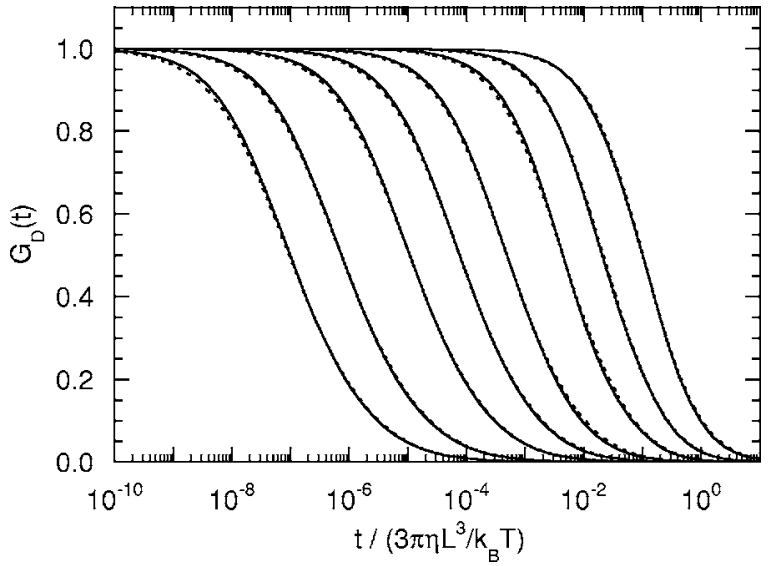

FIG. 11. Comparison of the full theoretical FCS correlation function (solid lines) to the analytical approximation of Eq. (50) (dotted lines) for various polymer lengths. The time scales of the analytical approximations are multiplied by the following factors to match the time scales of the full numerical correlation functions: 1.0 ( $p L=0.0085), 0.95$ (0.017), 1.0 (0.035), 1.2 (0.085), 1.3 (0.17), 1.4 (0.35), 1.5 (0.85), and 1.7 (1.7) (left to right). (For the other parameters see Fig. 8.)

$$
G_{\mathrm{D}}(t)=\left\{\begin{array}{ll}
{\left[1+2 D t / r_{0}^{2}+0.825\left(\Gamma_{B} t\right)^{3 / 4}\right]^{-1},} & \Gamma_{B} t \ll 1 \\
\frac{\sqrt{f^{2}-1}\left(\Gamma_{B} t\right)^{-3 / 4}}{1.65 \arctan \left(\sqrt{f^{2}-1}\right)}, & \Gamma_{B} t, \frac{L}{r_{0}} \gg 1
\end{array} .\right.
$$

Hence, two regimes are predicted for $1 / G_{\mathrm{D}}-1$ with an exponent of $\frac{3}{4}$. The one for $\Gamma_{B} t \ll 1$ is not obtained by the exact correlation function (33), which rather yields an exponent close to unity for $t \rightarrow 0$. The reason is again that the cosine function of Eq. (46) cannot be approximated by unity for $\Gamma_{B} t \ll 1$. The exponent $\approx 0.65$ (see Fig. 10 ) is a consequence of the transition from the short time behavior to the long time behavior. Since $\arctan \left(\sqrt{f^{2}-1}\right) / \sqrt{f^{2}-1}$ is much smaller than unity, the intermediate slope is smaller than $\frac{3}{4}$.

As previously pointed out, all correlation functions approach a limiting curve at large chain lengths. The reason is the weak chain length dependence of $\Gamma_{B}$ [cf. Eq. (47)]. As soon as the intramolecular dynamics determines the correlation function, the logarithmic chain length dependence yields only small changes in the time scale.

All the theoretical results discussed are in agreement with experimental findings. Figure 12 provides an example of measurements of actin filaments of various lengths. For all samples, the experimental label densities are large enough to provide label density independent signals.

A drawback of the experimental synthesis of actin filaments is that their length is difficult to adjust. In general, polydisperse samples are produced. Thus, the actual length of a measured filament is not precisely known. The filament length for a theoretical curve is therefore determined such that the theoretical time scale (which is the only adjustable parameter) matches the experimental data using the parameters $l_{p}=16 \mu \mathrm{m}, d=8 \mathrm{~nm}, \quad T=300 \mathrm{~K}, \quad \eta=0.01 \mathrm{P}, \quad r_{0}$ $=0.2 \mu \mathrm{m}$, and $f=7$. By this procedure, we obtain the follow-

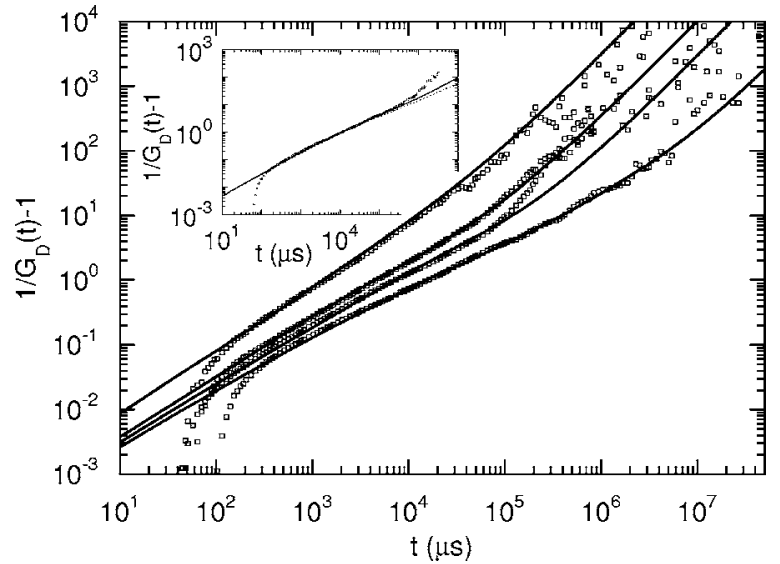

FIG. 12. Comparison of the theoretical FCS correlation functions (solid lines) to experimental measurements (squares) on actin filaments for $p L=0.0036,0.018,0.035$, and 0.15 (left to right). Inset: FCS correlation function for $p L=0.85$. The dotted line corresponds to the intramolecular contribution only.

ing lengths: $p L=0.0036 \quad(L=180 \mathrm{~nm}), \quad p L=0.018$ $(L=900 \mathrm{~nm}), \quad p L=0.035 \quad(L=1.75 \mu \mathrm{m}), \quad$ and $\quad p L=0.15$ $(L=7.5 \mu \mathrm{m})$. Note, for the calculation of $p$, we use $p=\left(3 l_{p}\right)^{-1}$, which is an adequate expression for the stiff actin filaments under consideration as shown in Ref. [26]. Considering the fact that the samples are polydisperse, the agreement is very good between the theoretical curves and the experimental data. For most of the measurements, the experimental data are well reproduced over the whole range of the experimentally accessible time scale.

The inset of Fig. 12 shows the limiting behavior for very long actin filaments $(L=43 \mu \mathrm{m}, p L=0.85)$. The exponent of the theoretical curve is approximately 0.67 compared to $\approx 1$ for $p L=0.0085$, which demonstrates that it decreases with increasing chain length.

The agreement between the theoretical expression and the experimental data also implies that the theoretically determined diffusion coefficients are close to those of actin filaments in solution.

\section{SUMMARY AND CONCLUSIONS}

In this paper, the fluorescence correlation function of semiflexible polymers has been determined theoretically. Moreover, measurements of the FCS correlation functions of DNA molecules and actin filaments of various lengths have been presented and compared to theoretical predictions.

For polymer radii of gyration smaller than the size of the focal volume, the decay of the FCS correlation function is determined by the diffusion of the whole molecule. When the typical chain dimension, e.g., the radius of gyration, exceeds the size of the focal volume, the intramolecular dynamics becomes relevant and can even dominate the relaxation behavior. However, the actual polymer length depends on its flexibility. Evidently, flexible polymers have to be much longer than semiflexible ones. Moreover, the longest relaxation time of the polymer has to be larger than the time at which the experimental FCS correlation function has essen- 
tially decayed in order to observe the internal dynamics. Increasing the chain length, this limit is easier reached for semiflexible polymers, because the relaxation time of a flexible polymer increases as $L^{2 / 3}$, whereas semiflexible polymers exhibit the much stronger dependence $L^{4}$. Hence, it is much easier to observe the intramolecular dynamics for the latter molecules.

Our calculations provide analytical approximations for the FCS correlation functions of flexible and semiflexible polymers. The comparison to the numerical solution of the full expression exhibits very good agreement in the limits of the applicability of the approximations.

Moreover, we find very good agreement between the experimental measurements and the theoretical calculations for DNA molecules and actin filaments of various lengths. The theoretical model takes hydrodynamic interactions into account via a preaveraged Rotne-Prager tensor. The agreement between measurements on double-stranded DNA and theoretical results suggests that hydrodynamic interactions are taken into account reasonably well. In particular, we cannot describe the experimental data by a Rouse-type approach, which neglects hydrodynamic interactions. This is in contrast to Ref. [59], where agreement with measurements on single labeled ds-DNA could only be obtained for the Rouse model. For highly labeled DNA molecules, a clear distinction between Rouse and Zimm dynamics is difficult and depends on chain length because the intramolecular dynamics yields only a small contribution to the decay of the correlation function. Diffusion coefficients, however, exhibit a strong dependence on hydrodynamic interactions and the observed quantitative agreement between our diffusion coefficients and those obtained by other measurements (cf. Sec. IV A 1) clearly underlines that hydrodynamic interactions are of major importants for DNA in solution. We would like to point out that the intramolecular contribution to the decay of the correlation function is more pronounced (cf. Fig. 7) for weakly labeled molecules. From our considerations, it is not at all obvious why Rouse-like dynamics of ds-DNA was observed in [59]. The clarification of this point requires more measurements on single labeled DNA molecules.
[1] D. Magde, E. Elson, and W. W. Webb, Phys. Rev. Lett. 29, 705 (1972).

[2] E. Elson and D. Magde, Biopolymers 13, 1 (1974).

[3] N. Thompson, in Topics in Fluorescence Spectroscopy, edited by J. R. Lakowicz (Plenum Press, New York, 1991), pp. 337378.

[4] M. Eigen and R. Rigler, Proc. Natl. Acad. Sci. U.S.A. 91, 5740 (1994).

[5] S. Maiti, U. Haupts, and W. W. Webb, Proc. Natl. Acad. Sci. U.S.A. 94, 11753 (1997).

[6] J. Enderlein, Phys. Rev. Lett. 83, 3804 (1999).

[7] O. Krichevsky and G. Bonnet, Rep. Prog. Phys. 65, 251 (2002).

[8] N. L. Thompson, A. M. Lieto, and N. W. Allen, Curr. Opin. Struct. Biol. 12, 634 (2002).

[9] J. Rička and T. Binkert, Phys. Rev. A 39, 2646 (1989).

[10] S. A. Sukhishvili, Y. Chen, J. D. Müller, E. Gratton, K. S. Schweizer, and S. Granick, Nature (London) 406, 146 (2000).

[11] H. Zettl, W. Häfner, A. Böker, H. Schmalz, M. Lanzendörfer, A. H. E. Müller, and G. Krausch, Macromolecules 37, 1917 (2004)

[12] J. Zhao and S. Granick, J. Am. Chem. Soc. 126, 6242 (2004).

[13] J. C. Politz, E. S. Brown, D. E. Wolf, and T. Pederson, Proc. Natl. Acad. Sci. U.S.A. 95, 6043 (1998).

[14] R. Brock, M. Hink, and T. Jovin, Biophys. J. 75, 2547 (1998).

[15] P. Schwille, U. Haupts, S. Maiti, and W. W. Webb, Biophys. J. 77, 2251 (1999).

[16] D. Lumma, S. Keller, T. Vilgis, and J. O. Rädler, Phys. Rev. Lett. 90, 218301 (2003).

[17] R. Köhler, P. Schwille, W. W. Webb, and M. Hanson, J. Cell. Sci. 113, 3921 (2000).

[18] P. Dittrich, F. Malvezzi-Campeggi, M. Jahnz, and P. Schwille, Biol. Chem. 383, 491 (2001).

[19] M. Kinjo and R. Rigler, Nucleic Acids Res. 23, 1795 (1995).

[20] B. Rauer, E. Neumann, J. Widengren, and R. Rigler, Biophys. Chem. 58, 3 (1996).
[21] P. Schwille, J. Bieschke, and F. Oehlenschläger, Biophys. Chem. 66, 211 (1997).

[22] K. Starchev, J. Zhang, and J. Buffle, J. Colloid Interface Sci. 203, 189 (1998).

[23] O. Kratky and G. Porod, Recl. Trav. Chim. Pays-Bas 68, 1106 (1949).

[24] J. Wilhelm and E. Frey, Phys. Rev. Lett. 77, 2581 (1996).

[25] R. G. Winkler, P. Reineker, and L. Harnau, J. Chem. Phys. 101, 8119 (1994).

[26] R. G. Winkler, J. Chem. Phys. 118, 2919 (2003).

[27] P. E. Rouse, J. Chem. Phys. 21, 1272 (1953).

[28] F. Bueche, J. Chem. Phys. 22, 603 (1954).

[29] B. H. Zimm, J. Chem. Phys. 24, 269 (1956).

[30] M. Doi and S. F. Edwards, The Theory of Polymer Dynamics (Clarendon Press, Oxford, 1986).

[31] A. Z. Akcasu, M. Benmouna, and C. C. Han, Polymer 21, 866 (1980).

[32] S. R. Aragón, Macromolecules 20, 370 (1987).

[33] R. Götter, K. Kroy, E. Frey, M. Bärmann, and E. Sackmann, Macromolecules 29, 30 (1996).

[34] K. Kroy and E. Frey, Phys. Rev. E 55, 3092 (1997).

[35] L. Harnau, R. G. Winkler, and P. Reineker, J. Chem. Phys. 102, 7750 (1995).

[36] L. Harnau, R. G. Winkler, and P. Reineker, J. Chem. Phys. 104, 6355 (1996).

[37] R. G. Winkler, L. Harnau, and P. Reineker, Macromol. Theory Simul. 6, 1007 (1997).

[38] L. Harnau, R. G. Winkler, and P. Reineker, J. Chem. Phys. 106, 2469 (1997).

[39] L. Harnau, R. G. Winkler, and P. Reineker, Europhys. Lett. 45, 488 (1999).

[40] R. G. Winkler, Phys. Rev. Lett. 82, 1843 (1999).

[41] D. Magde, E. Elson, and W. Webb, Phys. Rev. Lett. 29, 705 (1972).

[42] E. Elson and D. Magde, Biopolymers 13, 1 (1974).

[43] D. Magde, E. Elson, and W. Webb, Biopolymers 13, 29 
(1974).

[44] R. Rigler, U. Mets, J. Widengren, and P. Kask, Eur. Biophys. J. 22, 169 (1993).

[45] S. Hess and W. Webb, Biophys. J. 83, 2300 (2002).

[46] J. Rička and T. Binkert, Phys. Rev. A 39, 2646 (1989).

[47] J. Widengren, U. Mets, and R. Rigler, J. Phys. Chem. 99, 13368 (1995).

[48] J. Widengren, in Fluorescence Correlation Spectroscopy, edited by R. Rigler and E. Elson (Springer, New York, 2001), pp. 276-301.

[49] R. G. Winkler and P. Reineker, Macromolecules 25, 6891 (1992).

[50] J. Rotne and S. Prager, J. Chem. Phys. 50, 4831 (1969).

[51] H. Yamakawa, J. Chem. Phys. 53, 436 (1970).
[52] S. R. Aragón and R. Pecora, Macromolecules 18, 1868 (1985).

[53] G. Allegra and F. Ganazzoli, J. Chem. Phys. 74, 1310 (1981).

[54] E. Farge and A. C. Maggs, Macromolecules 26, 5014 (1993).

[55] T. Jankowski and R. Janka, in Fluorescence Correlation Spectroscopy, edited by R. Rigler and E. Elson (Springer, New York, 2001), pp. 331-345.

[56] R. Pecora, J. Chem. Phys. 48, 4126 (1968).

[57] R. Pecora, J. Chem. Phys. 49, 1032 (1968).

[58] D. E. Smith, T. T. Perkins, and S. Chu, Macromolecules 29, 1372 (1996).

[59] R. Shusterman, S. Alon, T. Gavrinyov, and O. Krichevsky, Phys. Rev. Lett. 92, 048303 (2004).

[60] L. Le Goff, O. Hallatschek, E. Frey, and F. Amblard, Phys. Rev. Lett. 89, 258101 (2002). 